\title{
GLOBAL EXISTENCE OF SOLUTIONS TO THE EINSTEIN EQUATIONS WITH COSMOLOGICAL CONSTANT FOR A PERFECT RELATIVISTIC FLUID ON A BIANCHI TYPE-I SPACE-TIME*
}

\author{
NORBERT NOUTCHEGUEME ${ }^{\dagger}$ AND L.R. GADJOU TAMGHE T $^{\ddagger}$
}

\begin{abstract}
Global existence is proved in the case of positive cosmological constant, and asymptotic behavior is investigated.
\end{abstract}

Key words. differential systems, local existence, global existence, constraints, asymptotic behavior

\section{Introduction}

Global dynamics of relativistic plasmas remains an open research area in General Relativity. In the relativistic kinetic theory, a "plasma" is a collection of fast-moving particles of several species evolving under the action of self-created natural forces. Such physical situations arise for instance in some media at very high temperature such as in burning reactors, nebular galaxies, solar winds, etc., where massive particles of ionized gas evolve with very high velocities under the action of both their common gravitational field and self-created electromagnetic forces. Physicists are now convinced that $99 \%$ of matter is at the state of plasma.

In this paper, we study the global evolution of an uncharged perfect fluid of pure radiation type, the background space-time being a Bianchi type-I space-time, which is a generalization of the Robertson-Walker space-time, considered to be the basic spacetime in cosmology, where homogeneous phenomena such as the one we consider here are relevant. The evolution is governed by the Einstein equations for the gravitational field. Our motivation for considering the cosmological constant $\Lambda$ is twofold: from the point of view of astrophysics, present measurements based on previous works with $\Lambda>0$ show that data on very distant astronomical objects such as supernovae of type$I_{a}$ can be determined precisely; from a mathematical point of view, previous works with $\Lambda>0$ show that the expansion of the universe is accelerating at late times. For more details on the cosmological constant, see [2].

The Einstein theory stipulates that the gravitational field, which in the case we consider depends on two real-valued functions $a$ and $b$, of the single variable $t$, is determined, through the Einstein equations, by the material content of the spacetime, which in our case is represented by a stress-matter tensor defined by the matter density $\rho$. The Einstein equations, coupled to the conservation laws, turn out to be a differential system to determine $a, b$, and $\rho$.

The Einstein theory stipulates that the gravitational field, which in the case we consider depends on two real-valued functions $a$ and $b$ of a single variable $t$, is determined through the Einstein equations coupled to the conservation laws, which turns out to be a differential system to determine $a, b$ and the matter density $\rho$.

We show that if $\Lambda<0$, there can exist no global solution. We show that if $\Lambda \geq 0$ then by a suitable change of variables and choice of initial data, there exists a global

\footnotetext{
*Received: April 4, 2008; accepted (in revised version): April 21, 2008.

${ }^{\dagger}$ Department of Mathematics, Faculty of Science, University of Yaounde I, P.O.B. 812 Yaounde, Cameroon (nnoutch@justice.com).

${ }^{\ddagger}$ Department of Mathematics, Faculty of Science, University of Yaounde I, P.O.B. 812 Yaounde, Cameroon (lgadjou@yahoo.fr).
} 
solution. Moreover, we show, by studying the asymptotic behavior, that the spacetime tends to the vacuum at later times, regardless to the size of the initial data. The present work differs from [3] in the sense that here we consider the Einstein equations with the cosmological constant, and we don't consider the aspect distribution of the matter.

The paper is organized as follows: In Section 2, we state the Einstein equations with cosmological constant as in [1], and we establish the differential system in $a, b$ and $\rho$. We also give some preliminary results. In Section 3, we prove global existence and we specify some cases where there cannot exist global solutions. In Section 4, we investigate the asymptotic behavior in the case of global existence.

\section{Equations and preliminary results}

Unless otherwise specified, Greek indices $\alpha, \beta, \gamma \ldots$, range from 0 to 3 and Latin indices $i, j, k \ldots$, range from 1 to 3 . We adopt the Einstein summation convention:

$$
a_{\alpha} b^{\alpha} \equiv \sum_{\alpha} a_{\alpha} b^{\alpha} \text {. }
$$

2.1. The Einstein equations. We consider the Bianchi type-I space-time $\left(\mathbb{R}^{4}, g\right)$ and we denote by $x^{\alpha}=\left(x^{0}, x^{i}\right)$ the usual coordinates in $\mathbb{R}^{4} ; g$ stands for the metric tensor of signature $(-,+,+,+)$ which can be written:

$$
g=-d t^{2}+a^{2}(t)\left(d x^{1}\right)^{2}+b^{2}(t)\left[\left(d x^{2}\right)^{2}+\left(d x^{3}\right)^{2}\right],
$$

where $a>0$ and $b>0$ are unknown functions of the single variable $t=x^{0}$. The Einstein equations for the metric tensor $g=\left(g_{\alpha \beta}\right)$, can be written, following [1]:

$$
R_{\alpha \beta}-\frac{1}{2} g_{\alpha \beta} R+\Lambda g_{\alpha \beta}=8 \pi T_{\alpha \beta}
$$

where $R_{\alpha \beta}$ is the Ricci tensor, contracted from the curvature tensor, $R=g^{\alpha \beta} R_{\alpha \beta}$ is the scalar curvature, contracted of the Ricci tensor, $\Lambda$ is a constant called the cosmological constant, and $T_{\alpha \beta}$ is the stress matter tensor, which represents the material content of space-time.

The general expression of the stress matter tensor of a relativistic perfect fluid is, in the chosen signature of $g$ :

$$
T_{\alpha \beta}=(\rho+p) u_{\alpha} u_{\beta}+p g_{\alpha \beta},
$$

where $\rho \geq 0$ and $p \geq 0$ are unknown functions of $t$ representing respectively the matter density and the pressure.

We consider a perfect fluid of pure radiation type, which means that $p=\frac{\rho}{3}$. The matter tensor then can be written as:

$$
T_{\alpha \beta}=\frac{4}{3} \rho u_{\alpha} u_{\beta}+\frac{1}{3} \rho g_{\alpha \beta},
$$

where $u=\left(u^{\alpha}\right)$ is a unit vector tangent to the geodesics of $g$.

In order to simplify, we consider a frame in which the fluid is spatially at rest. This implies $u^{i}=u_{i}=0$. Recall that indices are raised and lowered using the usual rules: $V^{\alpha}=g^{\alpha \beta} V_{\beta} ; V_{\alpha}=g_{\alpha \beta} V^{\beta},\left(g^{\alpha \beta}\right)$ standing for the inverse matrix of $\left(g_{\alpha \beta}\right)$.

Solving the Einstein equations (2.2) consists of determining, on one hand, the gravitational field represented by the metric tensor $g=\left(g_{\alpha \beta}\right)$ defined by the two unknown functions $a>0$ and $b>0$, and, on the other hand, its source represented by the matter density $\rho$. 
We deduce from the expression of $g$ given by (2.1) that:

$$
\left\{\begin{array}{l}
g_{00}=-1, g_{11}=a^{2}, g_{22}=g_{33}=b^{2} \\
g^{00}=-1, g^{11}=\frac{1}{a^{2}}, g^{22}=g^{33}=\frac{1}{b^{2}} \\
g_{\alpha \beta}=g^{\alpha \beta}=0 \quad \text { if } \quad \alpha \neq \beta
\end{array}\right.
$$

the Christoffel symbols of $g$ are given by:

$$
\Gamma_{\alpha \beta}^{\lambda}=\frac{1}{2} g^{\lambda \mu}\left[\partial_{\alpha} g_{\mu \beta}+\partial_{\beta} g_{\alpha \mu}-\partial_{\mu} g_{\alpha \beta}\right] .
$$

Recall that $\Gamma_{\alpha \beta}^{\lambda}=\Gamma_{\beta \alpha}^{\lambda}$. Expression (2.4) of $g_{\alpha \beta}$ and $g^{\alpha \beta}$ shows that the only nonvanishing Christoffel symbols are $\Gamma_{i 0}^{i}$ and $\Gamma_{i i}^{0}, i=1,2,3$. A direct calculation, using (2.4) and (2.5) gives:

$$
\left\{\begin{array}{l}
\Gamma_{10}^{1}=\frac{\dot{a}}{a}, \Gamma_{20}^{2}=\Gamma_{30}^{3}=\frac{\dot{b}}{b}, \Gamma_{11}^{0}=\dot{a} a, \Gamma_{22}^{0}=\Gamma_{33}^{0}=\dot{b} b \\
\Gamma_{\alpha \beta}^{\lambda}=0 \quad \text { otherwise }
\end{array}\right.
$$

where the dot stands for differentiation with respect to $t$.

The components of the Ricci tensor $R_{\alpha \beta}$ are given by

$$
R_{\alpha \beta}=\left(\partial_{\lambda} \Gamma_{\beta \alpha}^{\lambda}-\partial_{\beta} \Gamma_{\lambda \alpha}^{\lambda}\right)+\left(\Gamma_{\lambda \sigma}^{\lambda} \Gamma_{\alpha \beta}^{\sigma}-\Gamma_{\beta \sigma}^{\lambda} \Gamma_{\lambda \alpha}^{\sigma}\right) .
$$

Hence, using (2.4),(2.6),(2.7), $T_{00}=\rho, T_{11}=\frac{1}{3} \rho a^{2}, T_{22}=T_{33}=\frac{1}{3} \rho b^{2}, T_{\alpha \beta}=0$, if $\alpha \neq \beta$, the Einstein equations (2.2) give the following system in $a, b$ :

$$
\begin{aligned}
\left(\frac{\dot{b}}{b}\right)^{2}+2 \frac{\dot{a}}{a} \frac{\dot{b}}{b}-\Lambda & =8 \pi \rho \\
2 \frac{\ddot{b}}{b}+\left(\frac{\dot{b}}{b}\right)^{2}-\Lambda & =-\frac{8}{3} \pi \rho \\
\frac{\ddot{a}}{a}+\frac{\ddot{b}}{b}+\frac{\dot{a}}{a} \frac{\dot{b}}{b}-\Lambda & =-\frac{8}{3} \pi \rho .
\end{aligned}
$$

\subsection{Determination of $\rho$.}

PRoposition 2.1.

$$
\rho=\rho(0)\left(\frac{a(0) b(0)^{2}}{a b^{2}}\right)^{\frac{4}{3}} .
$$

Proof. We use the conservation laws $\nabla_{\alpha} T^{\alpha \beta}=0$, where $\nabla_{\alpha}$ is the usual covariant derivative in $g$. Expression (2.3) for $T^{\alpha \beta}$, where indices are raised by $g$, then gives, using the transparency of $g^{\alpha \beta}$ :

$$
\begin{array}{r}
\nabla_{\alpha}\left(\frac{4}{3} \rho u^{\alpha} u^{\beta}+\frac{1}{3} \rho g^{\alpha \beta}\right)=4 \nabla_{\alpha}\left(\rho u^{\alpha} u^{\beta}\right)+g^{\alpha \beta} \nabla_{\alpha} \rho=0, \\
4\left[u^{\beta} \nabla_{\alpha}\left(\rho u^{\alpha}\right)+\rho u^{\alpha} \nabla_{\alpha} u^{\beta}\right]+g^{\alpha \beta} \nabla_{\alpha} \rho=0 .
\end{array}
$$

Now, differentiating $u_{\beta} u^{\beta}=-1$ yields $u_{\beta} \nabla_{\alpha} u^{\beta}=0$. The contracted multiplication of (2.12) by $u_{\beta}$ then gives $4\left(-\nabla_{\alpha}\left(\rho u^{\alpha}\right)\right)+u^{\alpha} \nabla_{\alpha} \rho=0$, i.e., using (2.6):

$$
-4\left[\partial_{\alpha}\left(\rho u^{\alpha}\right)+\Gamma_{\alpha \lambda}^{\alpha} \rho u^{\alpha}\right]+u^{\alpha} \nabla_{\alpha} \rho=-4\left(\partial_{0}\left(\rho u^{0}\right)+\Gamma_{i 0}^{i} \rho u^{0}\right)+u^{0} \partial_{0} \rho=0,
$$


i.e.,

$$
-4 \partial_{0}\left(\rho u^{0}\right)-4\left(\frac{\dot{a}}{a}+2 \frac{\dot{b}}{b}\right) \rho u^{0}+u^{0} \partial_{0} \rho=0 .
$$

But $u=\left(u^{\alpha}\right)$ is a unit vector, then $u_{\beta} u^{\beta}=-1$; since $u^{i}=u_{i}=0$, we have $\left(u^{0}\right)^{2}=$ $\left(u_{0}\right)^{2}=1$, so $u^{0}$ is constant and different from 0 . Simplifying $(2.13)$ by $u^{0} \neq 0$ gives:

$$
\partial_{0} \rho+A(t) \rho=0
$$

where:

$$
A(t)=\frac{4}{3}\left(\frac{\dot{a}}{a}+2 \frac{\dot{b}}{b}\right)(t) .
$$

Then, multiply (2.14) by $\exp \left(\int_{0}^{t} A(s) d s\right)$, integrate over $[0, t]$, and use (2.15) to obtain (2.11).

2.3. The Cauchy problem. The system (2.8)-(2.9)-(2.10) is a system of second order non-linear differential equations in $a$ and $b$. Also consider the expression (2.11) for $\rho$. We suppose that $a_{0}>0, b_{0}>0, \dot{a}_{0} \in \mathbb{R}$, where $\dot{b}_{0} \in \mathbb{R}$ and $\rho_{0} \geq 0$, are given real numbers, and we look for solutions $a, b, \rho$ of the Einstein equations satisfying:

$$
a(0)=a_{0} ; b(0)=b_{0} ; \dot{a}(0)=\dot{a}_{0} ; \dot{b}(0)=\dot{b}_{0} ; \rho(0)=\rho_{0} .
$$

Our aim is to prove the global existence of solutions on $[0,+\infty$ [ of the above Cauchy problem; i.e., we are looking for global solutions satisfying the initial conditions (2.16). The values prescribed at $t=0$ will be called initial data. (2.11) shows that the matter density $\rho$ will be determined by:

$$
\rho=\rho_{0}\left(\frac{a_{0} b_{0}^{2}}{a b^{2}}\right)^{\frac{4}{3}},
$$

which shows that $\rho$ is known once $a$ and $b$ are known.

It is well-known that the Einstein equation (2.8), called the Hamiltonian constraint, is satisfied over the entire domain of the solutions $a, b, \rho$ if and only if (2.8) is satisfied for $t=0$, i.e., if the initial data $a_{0}, b_{0}, \dot{a}_{0}, \dot{b}_{0}, \rho_{0}$ satisfy the initial constraint

$$
\left(\frac{\dot{b}_{0}}{b_{0}}\right)^{2}+2 \frac{\dot{a}_{0}}{a_{0}} \frac{\dot{b}_{0}}{b_{0}}-\Lambda=8 \pi \rho_{0} .
$$

REMARK 2.2. (2.9) and (2.10) are called the evolution equations, and in what follows, we suppose that the initial data satisfy (2.18). We will then use the Hamiltonian constraint (2.8) as a property of the solutions.

\section{Global existence of solutions}

3.1. Change of variables and preliminary properties. In order to have a differential system of first order, we set:

$$
u=\frac{\dot{a}}{a} ; \quad v=\frac{\dot{b}}{b} .
$$


Then $\dot{u}=\frac{\ddot{a}}{a}-u^{2} ; \dot{v}=\frac{\ddot{b}}{b}-v^{2}$. Hence, the evolution equations (2.9), (2.10) can be written in terms of $u$ and $v$ :

$$
\begin{aligned}
2 \dot{v}+3 v^{2}-\Lambda & =-\frac{8}{3} \pi \rho, \\
\dot{u}+\dot{v}+u^{2}+v^{2}+u v-\Lambda & =-\frac{8}{3} \pi \rho .
\end{aligned}
$$

We look for solutions $(u, v)$ for (3.2)-(3.3) satisfying at $t=0$, and following the change of variables (3.1):

$$
u(0)=u_{0} ; \quad v(0)=v_{0} ; \quad \text { with }: \quad u_{0}=\frac{\dot{a}_{0}}{a_{0}} ; v_{0}=\frac{\dot{b}_{0}}{b_{0}} .
$$

Notice that the system $(2.9)-(2.10)$ in $(a, b)$ and the system (3.2)-(3.3) in $(u, v)$ are equivalent in the sense that (2.9)-(2.10) imply (3.2)-(3.3), and, conversely, the knowledge of $u=\frac{\dot{a}}{a}$ and $v=\frac{\dot{b}}{b}$ gives $a, b$ by direct integration.

We will need the Hamiltonian constraint (2.8) which in terms of $u$ and $v$ is

$$
v^{2}+2 u v-\Lambda=8 \pi \rho .
$$

Now we eliminate $\rho$ from the above equations.

First multiply (3.2) by 3 , add the result to (3.5) and deduce $\dot{v}$; next, subtracting (3.2) from (3.3) and using the value of $\dot{v}$ given by the previous operation yields the system:

$$
\begin{aligned}
& \dot{u}=\frac{1}{3} v^{2}-u^{2}-\frac{4}{3} u v+\frac{2}{3} \Lambda, \\
& \dot{v}=-\frac{5}{3} v^{2}-\frac{1}{3} u v+\frac{2}{3} \Lambda .
\end{aligned}
$$

We will study system $(3.6)-(3.7)$ in $(u, v)$ with the initial data (3.4). We first establish some properties.

Subtracting (3.2) from (3.3) gives:

$$
(\dot{u}-\dot{v})+(u-v)(u+2 v)=0 .
$$

Solving this as an ordinary differential equation (ODE) in $(u-v)$ gives:

$$
u-v=\left(u_{0}-v_{0}\right) \exp \left(-\int_{0}^{t}(u+2 v)(s) d s\right),
$$

which shows that $u-v$ has the constant sign of $u_{0}-v_{0}$. Next, evaluating $\Lambda$ in (3.5) gives $\frac{2}{3} \Lambda=\frac{2}{3}\left(v^{2}+2 u v-8 \pi \rho\right)$, which, substituted in (3.6) and (3.7) gives, using $\rho \geq 0$, the inequalities

$$
\begin{aligned}
& \dot{u} \leq-(u-v)(u+v), \\
& \dot{v} \leq v(u-v) .
\end{aligned}
$$

Next, we prove:

LEMMA 3.1. Let $(u, v)$ be a solution of (3.4)-(3.6)-(3.7) such that $u\left(t_{0}\right)=0$ for some $t_{0} \geq 0$. Suppose $\Lambda \geq 0$, and, in the case $\Lambda=0$, suppose in addition that $u_{0} \neq v_{0}$. Then $u$ is increasing in a neighborhood of $t_{0}$, and $u \geq 0$ if $u_{0}>0$. 
Proof. Evaluating (3.6) at $t_{0}$ gives, since $u\left(t_{0}\right)=0: \dot{u}\left(t_{0}\right)=\frac{1}{3} v^{2}\left(t_{0}\right)+\frac{2}{3} \Lambda$; then, if $\Lambda>0$, we have $\dot{u}\left(t_{0}\right) \geq \frac{2}{3} \Lambda>0$; if $\Lambda=0$; then $\dot{u}\left(t_{0}\right)=\frac{1}{3} v^{2}\left(t_{0}\right)$; by hypothesis, we suppose in this case that $u_{0} \neq v_{0} ;(3.8)$ then implies $v^{2}\left(t_{0}\right) \neq u^{2}\left(t_{0}\right)=0$. So we have, in all cases that $\dot{u}\left(t_{0}\right)>0$. Now the solution $u$ is of class $C^{1}$, i.e. $\dot{u}$ is continuous. The continuity of $\dot{u}$ at $t=t_{0}$ implies that $\exists \delta>0$ such that $\left|t-t_{0}\right|<\delta \Rightarrow\left|\dot{u}(t)-\dot{u}\left(t_{0}\right)\right|<$ $\frac{\dot{u}\left(t_{0}\right)}{2}$; hence, $\left|t-t_{0}\right|<\delta \Rightarrow \dot{u}(t)>\frac{\dot{u}\left(t_{0}\right)}{2}>0$, which implies $\dot{u}>0$ over the neighborhood $\left.I_{\delta}=\right] t_{0}-\delta, t_{0}+\delta\left[\right.$ of $t_{0}$. Hence, $u$ is increasing over $I_{\delta}$.

Let $t_{0} \geq 0$ be any point such that $u\left(t_{0}\right)=0$. By 1$)$, there exists $\delta>0$ such that $\left(t_{0} \leq t<t_{0}+\delta\right) \Rightarrow\left(u(t) \geq u\left(t_{0}\right)=0\right)$. We then conclude that, if $u(0)=u_{0}>0$, then $u$ remains positive.

We end this section by recalling the following well-known result:

LEMMA 3.2. Let $x$ and $y$ be 2 real-valued differentiable functions of $t$ satisfying:

$$
\left\{\begin{array}{l}
\dot{x} \leq-\alpha x^{2} \\
\dot{y}=-\alpha y^{2} \\
x\left(t_{0}\right)=y\left(t_{0}\right)
\end{array}\right.
$$

where $\alpha>0$ is a constant and $t_{0}$ a given value of $t$. Then:

$$
x(t) \leq y(t) \quad \text { for } \quad t \geq t_{0} .
$$

REMARK 3.3. The initial constraint (2.18) may also be written as

$$
\left(\frac{\dot{a_{0}}}{a_{0}}+\frac{\dot{b_{0}}}{b_{0}}\right)^{2}=\Lambda+8 \pi \rho_{0}+\left(\frac{\dot{a_{0}}}{a_{0}}\right)^{2}
$$

which implies, since the left hand side is positive:

$$
\Lambda \in\left[-8 \pi \rho_{0}-\left(\frac{\dot{a_{0}}}{a_{0}}\right)^{2},+\infty[.\right.
$$

By the classical theory on first order differential systems, the Cauchy problem for the system (3.6)-(3.7) in $(u, v)$ has a unique local solution $(u, v)$. The problem we want to solve here is to prove whether or not this solution is global, i.e., defined over the whole interval $\left[0,+\infty\left[\right.\right.$. We suppose $\rho_{0}>0$ in what follows. Expression (2.17) of $\rho$ then implies $\rho>0$, since $a_{0}>0, b_{0}>0, a>0, b>0$.

3.2. Global existence of solutions. We first prove:

Proposition 3.4. If $\Lambda \in\left[-8 \pi \rho_{0}-\left(\frac{\dot{a_{0}}}{a_{0}}\right)^{2}, 0[\right.$, then there can exist no global solutions to the Einstein equations.

If $\Lambda \geq 0, v_{0}<0$ and $u_{0}>0$, then there can exist no global solutions to the Einstein equations.

Proof. Recall that the lower bound of $\Lambda$ is provided by (3.11). Suppose that there exists a global solution $(a, b), a>0, b>0$ on $[0,+\infty[$ to the Einstein equations. The evolution equations $(2.9),(2.10)$ show that $a$ and $b$ are of class $C^{2}$. Then the change of 
variables (3.1) shows that $u$ and $v$ are of class $C^{1}$ on $[0,+\infty[$. Now (3.2) gives, since $\rho \geq 0, \dot{v} \leq-\frac{3}{2} v^{2}+\Lambda$, and this implies, since $\Lambda<0$, that

$$
\begin{aligned}
& \dot{v} \leq-\frac{3}{2} v^{2}, \\
& \dot{v} \leq \Lambda .
\end{aligned}
$$

Integrating (3.13) over $[0, t], t \geq 0$ gives: $v(t) \leq v_{0}+\Lambda t$, which implies that

$$
v(t) \rightarrow-\infty \quad \text { when } \quad t \rightarrow+\infty .
$$

So there exists $t_{0}$ such that $v\left(t_{0}\right)<0$. Now let $w$ be a function satisfying

$$
\begin{gathered}
\dot{w}=-\frac{3}{2} w^{2}, \\
w\left(t_{0}\right)=v\left(t_{0}\right)<0 .
\end{gathered}
$$

Applying Lemma 3.2 with $\alpha=\frac{3}{2}$ to (3.12)-(3.14)-(3.15) gives:

$$
v(t) \leq w(t), \quad t \geq t_{0} .
$$

Equation (3.14) also shows that $\dot{w} \leq 0$; so $w$ is decreasing and hence

$$
w(t) \leq w\left(t_{0}\right)<0, \quad t \geq t_{0} .
$$

So $w$ does not vanish on $\left.]-\infty, t_{0}\right]$. Separating (3.14) and integrating over $\left[t_{0}, t\right], t \geq t_{0}$, gives:

$$
w(t)=\frac{\frac{2}{3}}{\frac{2}{3} \frac{1}{v\left(t_{0}\right)}+\left(t-t_{0}\right)} .
$$

But (3.17) shows that

$$
w(t) \rightarrow-\infty \quad \text { when } \quad t \rightarrow<t^{*}, \quad \text { where } \quad t^{*}=t_{0}-\frac{2}{3 v\left(t_{0}\right)}>t_{0}
$$

since $v\left(t_{0}\right)<0$. And (3.16) implies that $v(t) \rightarrow-\infty$ when $t \rightarrow<t^{*}$. But this doesn't

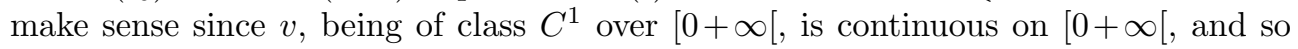
should remain bounded on every line segment such as $\left[t_{0}, t^{*}\right]$. We conclude that there cannot exist global solutions in the case $\Lambda<0$.

Also suppose that there exists a global solution $a>0, b>0$ to the Einstein equations and consider (3.1). The Hamiltonian constraint (3.5) written as

$$
v(v+2 u)=8 \pi \rho+\Lambda
$$

implies, since $\rho>0, \Lambda \geq 0$, that $v(v+2 u)>0$. This implies that $v$ cannot vanish. We then conclude, since $v$ is continuous that

$$
\left(v(0)=v_{0}<0\right) \Rightarrow(v<0) .
$$

Now we have $v_{0}<0<u_{0}$, so $u_{0} \neq v_{0}$, and by Lemma 3.1 , conculsion 2$) ;\left(u_{0}>0\right) \Rightarrow$ $(u \geq 0)$ The inequality (3.10) then implies:

$$
\dot{v} \leq v(u-v)=v u-v^{2} \leq-v^{2}
$$


so that we have:

$$
\left\{\begin{array}{l}
\dot{v} \leq-v^{2} \\
v<0
\end{array}\right.
$$

We then proceed as in 1), applying Lemma 3.3 with $\alpha=1$ to conclude that there can exist no global solution in this case.

THEOREM 3.5. Suppose $\Lambda \geq 0$. Then, if $v_{0}>0$, there exists a global solution to the Einstein equations.

Proof. We know that there exists a local solution $(u, v)$ to the system $(3.4)-(3.6)-$ (3.7). By the theory of first order differential systems, to show that this solution is global, it will be enough to prove that any solution of the Cauchy problem (3.4)(3.6)-(3.7) remains uniformly bounded.

Suppose $u_{0}<v_{0}$. Then, by 3.8,u<v. As we saw before, since $\rho>0$ and $\Lambda \geq 0$, we deduce from $(3.5)$ that $v(v+2 u)>0$. This implies that $v$ and $v+2 u$ do not vanish, and have the same sign. Since $v$ is continuous and doesn't vanish:

$$
\left(v(0)=v_{0}>0\right) \Rightarrow(v>0)
$$

But $v$ and $v+2 u$ have the same sign, so:

$$
v+2 u>0
$$

Now, since $v>0$ and $u<v$, the inequality (3.10) implies $\dot{v}<0$, so $v$ is decreasing and $v \leq v_{0}$. We then conclude, using (3.19), that:

$$
-\frac{v_{0}}{2}<u<v \leq v_{0}
$$

which shows that the solution $(u, v)$ remains bounded.

Suppose $u_{0} \geq v_{0}$. Then, by $3.8, u \geq v$. Since $v_{0}>0$, (3.18) implies

$$
0<v \leq u
$$

(3.20) shows that $v>0$ and $u>0$, hence $u+v>0$, and since $u-v \geq 0$, the inequality (3.9) implies $\dot{u} \leq 0$. So $u$ is decreasing, which implies that $u \leq u_{0}$. We then have finally:

$$
0<v \leq u \leq u_{0}
$$

which shows that the solution $(u, v)$ remains uniformly bounded. This completes the proof of Theorem 3.5.

\section{Asymptotic behavior}

We prove:

THEOREM 4.1. Suppose $\Lambda \geq 0$ and $0<u_{0} \leq v_{0}$. Then the space-time $\left(\mathbb{R}^{4}, g_{\alpha \beta}, T_{\alpha \beta}\right)$, which exists globally, tends to the vacuum at late times.

Proof. We have to prove that

$$
T_{\alpha \beta}(t) \longrightarrow 0 \quad \text { when } \quad t \rightarrow+\infty \text {. }
$$


Case $\Lambda>0$

The Hamiltonian constraint (2.8), written $\left(\frac{\dot{a}}{a}+\frac{\dot{b}}{b}\right)^{2}=8 \pi \rho+\Lambda+\left(\frac{\dot{a}}{a}\right)^{2}$, implies, since $\rho>0$, that

$$
\left(\frac{\dot{a}}{a}+\frac{\dot{b}}{b}\right)^{2} \geq \Lambda>0 .
$$

Now, since $\Lambda \neq 0$ and $0<u_{0} \leq v_{0}$, (3.8) and Lemma 3.1, conculsion 2), imply that $0 \leq u \leq v$, so we have that $u=\frac{\dot{a}}{a} \geq 0$ and $v=\frac{\dot{b}}{b} \geq 0$ and (4.1) gives

$$
\frac{\dot{a}}{a}+\frac{\dot{b}}{b} \geq \sqrt{\Lambda}
$$

since $\frac{\dot{a}}{a} \geq 0, \frac{\dot{b}}{b} \geq 0,(4.2)$ also implies for $\alpha, \beta$ constant:

$$
\alpha \frac{\dot{a}}{a}+\beta \frac{\dot{b}}{b} \geq \sqrt{\Lambda}, \quad \alpha \geq 1 \quad \beta \geq 1 .
$$

Integrating (4.3) over $[0, t], t \geq 0$ gives:

$$
a^{\alpha}(t) b^{\beta}(t) \geq a_{0}^{\alpha} b_{0}^{\beta} \exp (\sqrt{\Lambda} t),
$$

which shows an exponential growth in the case $\Lambda>0$, and implies:

$$
a^{\alpha}(t) b^{\beta}(t) \rightarrow+\infty \quad \text { when } \quad t \rightarrow+\infty ; \quad \text { with } \quad \alpha \geq 1 \quad \beta \geq 1 .
$$

Hence, expression (2.17) of $\rho$ shows, taking $\alpha=\frac{4}{3}, \beta=\frac{8}{3}$, that:

$$
\rho(t)=\rho_{0}\left(a_{0} b_{0}^{2}\right)^{\frac{4}{3}}\left(a^{-\frac{4}{3}} b^{-\frac{8}{3}}\right)(t) \rightarrow 0 \quad \text { when } \quad t \rightarrow+\infty .
$$

Then $T_{00}=\rho \rightarrow 0$ when $t \rightarrow+\infty$. For the common value of $T_{22}$ and $T_{33}$, taking $\alpha=4, \beta=2$ in (4.4):

$$
\begin{aligned}
T_{22} & =\frac{1}{3} \rho b^{2}(t)=\frac{1}{3} \rho_{0}\left(a_{0} b_{0}^{2}\right)^{\frac{4}{3}} b^{2} a^{-\frac{4}{3}} b^{-\frac{8}{3}}(t)=\frac{\rho_{0}}{3}\left(a_{0} b_{0}^{2}\right)^{\frac{4}{3}} a^{-\frac{4}{3}} b^{-\frac{2}{3}}(t) \\
& =\frac{1}{3} \rho_{0}\left(a_{0} b_{0}^{2}\right)^{\frac{4}{3}}\left(a^{4} b^{2}\right)^{-\frac{1}{3}} \rightarrow 0 \quad \text { when } \quad t \rightarrow+\infty
\end{aligned}
$$

Finally, since $\left(u_{0} \leq v_{0}\right) \Rightarrow(u \leq v)$, we have $\frac{\dot{a}}{a} \leq \frac{\dot{b}}{b}$; integrating over $[0, t], t \geq 0$, yields: $a(t) \leq \frac{a_{0}}{b_{0}} b(t)$. Proceeding, as in (4.5):

$$
T_{11}=\frac{1}{3} a^{2} \rho \leq \frac{1}{3}\left(\frac{a_{0}}{b_{0}}\right)^{2} b^{2} \rho(t) \longrightarrow 0 \quad \text { when } \quad t \rightarrow+\infty .
$$

Hence, in the case $\Lambda>0$,

$$
T_{\alpha \beta}(t) \rightarrow 0 \quad \text { when } \quad t \rightarrow+\infty
$$

Case $\Lambda=0$ 
First deduce from (3.6) $-(3.7)$ with $\Lambda=0$ that we have $(2 \dot{u}+\dot{v})+(2 u+v)(u+v)=0$ which, solved as first order ODE in $2 u+v$, gives:

$$
2 u+v=\left(2 u_{0}+v_{0}\right) \exp \left(-\int_{0}^{t}(u+v)(s) d s .\right)
$$

The global solution $(u, v)$ of (3.4)-(3.6)-(3.7) gives, integrating (3.1):

$$
a(t)=a_{0} \exp \left(\int_{0}^{t} u(s) d s\right) ; b(t)=b_{0} \exp \left(\int_{0}^{t} v(s) d s\right) .
$$

We know that $\left(0<u_{0} \leq v_{0}\right) \Rightarrow(0 \leq u \leq v)$, so that (4.7) gives:

$$
a b^{2}(t)=a_{0} b_{0}^{2} \exp \int_{0}^{t}(u+2 v)(s) d s ; \quad a \geq a_{0}>0 ; \quad b \geq b_{0}>0 .
$$

Now, taking the derivative of $\left(a b^{2}\right)$ and using $v \geq 0$, we have:

$$
\left(a b^{2}\right)^{\cdot}=(u+2 v) a b^{2}=\left[\frac{1}{2}(2 u+v)+\frac{3}{2} v\right] a b^{2} \geq \frac{1}{2}(2 u+v) a b^{2} .
$$

which gives, using (4.6),(4.8), and $v \geq 0$ :

$$
\left(a b^{2}\right) \geq \frac{1}{2}\left(2 u_{0}+v_{0}\right) a_{0} b_{0}^{2} \exp \left(\int_{0}^{t} v(s) d s\right) \geq \frac{1}{2}\left(2 u_{0}+v_{0}\right) a_{0} b_{0}^{2}:=k_{0}>0 .
$$

Then, integrating over $[0, t]$ yields $a b^{2}(t) \geq k_{0} t+a_{0} b_{0}^{2}$ which shows a slow growth in the case $\Lambda=0$ and implies that

$$
a b^{2}(t) \rightarrow+\infty \quad \text { when } \quad t \rightarrow+\infty .
$$

Now we have, using expression (2.17) for $\rho$ and (4.9) that

$$
T_{00}=\rho(t)=\rho_{0}\left(a_{0} b_{0}^{2}\right)^{\frac{4}{3}}\left(a b^{2}\right)^{-\frac{4}{3}}(t) \rightarrow 0 \quad \text { when } \quad t \rightarrow+\infty .
$$

Next we also have, using (2.17) and (4.8), which gives $\frac{1}{a} \leq \frac{1}{a_{0}}$, that

$$
\begin{gathered}
T_{22}=T_{33}=\frac{1}{3} \rho b^{2}(t)=\frac{1}{3} \rho_{0}\left(a_{0} b_{0}^{2}\right)^{\frac{4}{3}} b^{2}\left(a b^{2}\right)^{-\frac{4}{3}}=\frac{1}{3 a} \rho_{0}\left(a_{0} b_{0}^{2}\right)^{\frac{4}{3}}\left(a b^{2}\right)\left(a b^{2}\right)^{-\frac{4}{3}}(t), \\
T_{22}=T_{33}=\frac{1}{3} \rho b^{2}(t) \leq \frac{\rho_{0}}{3 a_{0}}\left(a_{0} b_{0}^{2}\right)^{\frac{4}{3}}\left(a b^{2}\right)^{-\frac{1}{3}}(t) \rightarrow 0 \quad \text { when } \quad t \rightarrow+\infty
\end{gathered}
$$

Finally, since $(u \leq v) \Rightarrow\left(\frac{a}{a_{0}} \leq \frac{b}{b_{0}}\right)$, and proceeding as for (4.10),

$$
T_{11}=\frac{1}{3} a^{2} \rho(t) \leq \frac{1}{3}\left(\frac{a_{0}}{b_{0}}\right)^{2} \rho b^{2}(t) \longrightarrow 0 \quad \text { when } \quad t \rightarrow+\infty .
$$

We then conclude that we also have, in the case $\Lambda=0$, that

$$
T_{\alpha \beta}(t) \rightarrow 0 \quad \text { when } \quad t \rightarrow+\infty .
$$

This completes the proof of Theorem 4.1. 


\section{REFERENCES}

[1] S.W. Hawking and F.R. Ellis, The Large Scale Structure of Space-Time, Cambrige Monographs on Math. Phys, CUP, 1973.

[2] N. Straumann, On the cosmological constant problems and the astronomical evidence for a homogeneous energy density with negative pressure, astro-ph/ 023330, preprint, 2002.

[3] N. Noutchegueme and D. Dongo, Global existence of solutions for the Einstein-Boltzmann system, in a Bianchi type I space-time, Class. Quantum. Grav., 23, 2979-3003, 2006. 